\title{
Análise da zona afetada pelo calor em soldagem dupla camada utilizando o método dos elementos finitos
}

\author{
Analysis of heat affected zone in double \\ layer welding using the finite element \\ method
} Adry Kleber Ferreira de Lima ${ }^{1}$

\footnotetext{
${ }^{1}$ Universidade Federal do Pará, UFPA/FEM, Br. 422 Km 13 s/n, Canteiro de Obras da UHE, Campus Universitário de Tucuruí, CEP 68464-000, Tucuruí, PA, Brasil.

${ }^{2}$ Universidade Federal de Santa Catarina, UFSC/FEM, R. Eng. Agronômico Andrei Cristian Ferreira s/n, Campus Reitor João Lima, CEP: 88040-900, Florianópolis, SC, Brasil.

e-mail: dgarcia@ufpa.br, rafael.bernardi@hotmail.com, adry@ufpa.br
}

\section{RESUMO}

A técnica de soldagem dupla camada utiliza método controlado de deposição, onde a relação entre os aportes térmicos da segunda para a primeira camada de solda (E2/E1) deve ser criteriosamente definida para promover o refino dos grãos da Zona Afetada pelo Calor (ZAC). Entretanto, a parametrização de processos de soldagem para a realização desta técnica tem sido de modo experimental, requerendo diversos ensaios, corpos de prova e consumíveis, demandando tempo e elevado custo. Uma possível solução para tal problemática é a análise computacional aplicada à soldagem, tornando o estudo desta de grande relevância. Este trabalho objetiva o estudo inicial do comportamento da ZAC em soldagem dupla camada utilizando o Método de Elementos Finitos (MEF). Experimentos práticos foram realizados para comparar e validar o modelo numérico. Dessa maneira, seis corpos de prova foram fabricados, três com camada simples e três com dupla camada. Foram utilizadas três energias de soldagem diferentes para os primeiros passes $(0.571,0.428$ e $0.342 \mathrm{~kJ} / \mathrm{mm})$ e o dobro de tais energias para o segundo passe (taxa de energia de 1:2). Comparou-se as macrografias dos resultados experimentais e numéricos, tanto para a camada simples quanto para a dupla camada, bem como realizou-se a medição das dimensões da ZAC ao longo da seção transversal dos corpos de prova. Dessa maneira, notou-se que a taxa de energia de 1:2 é suficiente para atingir a zona de grãos grosseiros da primeira camada. Ainda, os resultados macrográficos e as medições das ZAC apresentaram ótima convergência entre as análises experimental e numérica, validando o modelo proposto.

Palavras-chave: Distribuição de calor em soldagem. Simulação em soldagem. Sobreposição dos ciclos térmicos.

\section{ABSTRACT}

The double layer welding technique uses a controlled deposition method, where the relationship between the thermal inputs from the second layer to the first (E2/E1) must be carefully defined to promote grain refinement in the Heat Affected Zone (HAZ). However, the parameterization of the welding processes for this technique is usually experimental, requiring tests, samples and consumables, demanding time and high cost. A possible solution to this problem is a computational analysis applied to the welding process, making this study to be of great relevance. This work aims an initial study of HAZ behavior in double layer welding using the Finite Element Method (FEM). Experiments were performed to compare and validate the numerical model. In this way, six samples were made, three with a single layer and three with double layer. Three different welding energies were used for the first layer $(0.571,0.428$ and $0.342 \mathrm{~kJ} / \mathrm{mm})$ and the double of those energies for the second layer (1:2 energy rate). Macrographs of the experimental and numerical results were compared for single and double layer conditions, and HAZ dimensions were measured along the sample cross section. Therefore, it was noted that 1:2 energy rate is sufficient to reach the coarse grain zone of the first layer. In addition, macrographic and HAZ measurements results showed excellent convergence between the experimental and numerical analysis, validating the proposed model. 
Keywords: Heat distribution in welding. Welding simulation. Overlapping thermal cycles.

\section{INTRODUÇÃO}

A soldagem é um processo usado na fabricação de várias estruturas metálicas, com aplicações de seções finas a seções mais grossas, como vasos de pressão, plantas químicas e reatores nucleares. Os principais problemas associados à soldagem são a presença de tensões residuais e as transformações estruturais na junta soldada que podem causar falhas durante a operação [1]. Durante o processo de soldagem, a distribuição de temperatura não é uniforme levando a formação de três regiões: a zona de fusão, a zona afetada pelo calor (ZAC) e o metal base inalterado.

O deslocamento da fonte de calor sobre a superfície da junta gera, um gradiente de temperatura causando uma mudança complexa na morfologia microestrutural da zona afetada pelo calor (ZAC), e de suas propriedades mecânicas. As mudanças microestruturais no metal de solda e na ZAC ocorrem em função das taxas de aquecimento e resfriamento as quais, por sua vez, dependem da energia de soldagem, da espessura e geometria da peça, do pré-aquecimento e da temperatura interpasse [2]. Entre as diferentes regiões formadas na zona afetada pelo calor a região de crescimento dos grãos, grãos grosseiros, merece destaque, pois o crescimento dos grãos austeníticos aumenta a temperabilidade nesta região da ZAC, favorecendo a formação de fases de alta dureza (bainita e/ou martensita), ocasionando a suscetibilidade a trincas a frio. Entretanto, a formação de fases de baixa tenacidade na região da ZAC é dependente da composição química do metal de base, do ciclo térmico imposto e da taxa de resfriamento $[1,3,4]$.

Em soldagem multipasses a microestrutura da ZAC é ainda mais complexa devido aos diferentes ciclos térmicos, que causam a re-austenitização e subsequentes transformações no resfriamento e, assim possibilitando um refinamento parcial da sua microestrutura. Em função desse comportamento são possíveis reduções na tensão residual e melhoria na tenacidade. A passagem de cada ciclo térmico referente ao cordão de solda subsequente, refina os grãos (revenimento) de parte do cordão de solda anterior, resultando em grãos grosseiros recristalizados [3,4].

Os efeitos do revenimento pela sobreposição dos ciclos térmicos são geralmente benéficos para o desempenho e a vida útil das soldas multipasses, conforme sugerido por Aloraier et al. [5] e Rathod et al. [6]. Esse comportamento é particularmente interessante quando o tratamento térmico pós soldagem é muito difícil ou caro para executar em uma estrutura soldada. Para soldagem de reparo, Aloraier et al. [7] e Lant et al. [8] demonstraram que a otimização do processo de revenimento em uma soldagem multipasse é uma parte essencial no projeto de estrutura soldada. O calor resultante do cordão de solda anterior age como préaquecimento diminuindo a velocidade de resfriamento, que também atua para reduzir as tensões residuais [8].

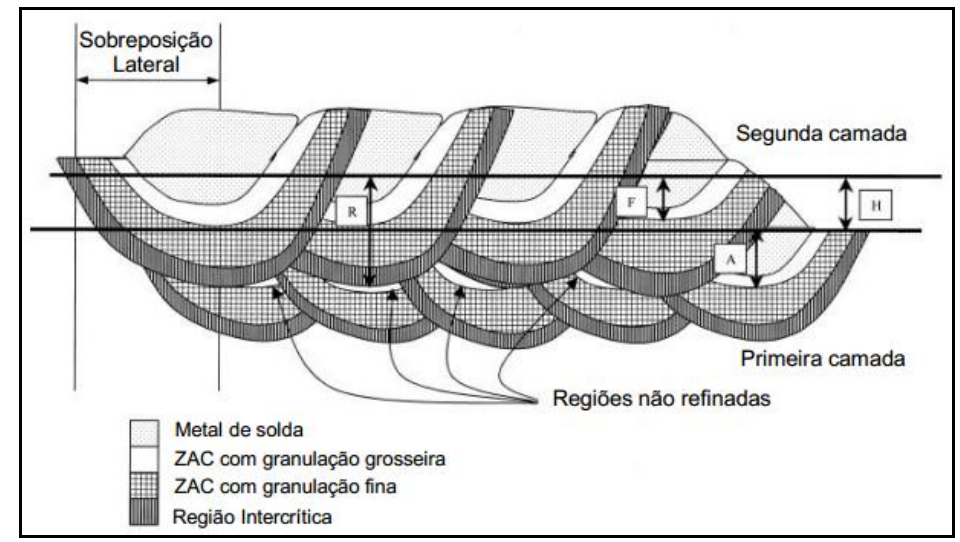

Figura 1: Representação ilustrativa da técnica da dupla camada, (F) penetração da segunda camada, (A) penetração da primeira camada, $(\mathrm{R})$ profundidade refinada pela segunda camada, $(\mathrm{H})$ altura média da primeira camada [8].

Alguns estudos realizados [8,9] apontaram que o calor gerado pela deposição da segunda camada pode refinar e/ou revenir a a região de grãos grosseiros da ZAC (ZACGG) da primeira camada, reduzindo a dureza e aumentando a tenacidade em materiais com elevado carbono equivalente, essa técnica é conhecida como dupla camada, Figura 1. O desempenho da técnica dupla camada de solda está relacionado à correta relação entre as energias de soldagem da primeira e da segunda camada, bem como do controle da temperatura inicial e interpasses, e uma adequada sobreposição dos cordões de solda. 
O emprego da simulação numérica em processo de soldagem cresceu nos últimos anos em função principalmente do aumento da capacidade dos computadores e à disponibilidade de códigos numéricos comerciais. Entretanto, muitas suposições e aproximações ainda são necessárias devido à complexidade dos fenômenos envolvidos nesse processo. Para Farias et. al. [10] a principal dificuldade da simulação térmica em um processo de soldagem é a modelagem da fonte de calor. Após o modelo de solução analítica proposto por Rosenthal [11] considerando uma fonte de calor pontual ou linear, outros modelos com diferentes distribuições de fonte de calor mais realistas foram propostos. Um modelo volumétrico clássico de fonte de calor é a distribuição elipsóide dupla, Figura 2, desenvolvida por Goldak [12].

Para Goldak [12], o estudo da soldagem computacional permite a redução de custos, já que há uma redução do número de experimentos, com uma boa margem de precisão. Segundo Goldak et. al. [13], a distribuição do fluxo de calor no arco deve ser estimada através de dados experimentais da junta soldada (zona fundida). A precisão do método depende da quantidade de nós e elementos, e do tamanho e tipo dos elementos presentes na malha. Um dos aspectos mais importantes do método de elementos finitos (MEF) diz respeito a sua convergência. Embora, seja um método aproximado, pode-se demonstrar que em uma malha consistente, na medida que o tamanho dos elementos finitos tende a zero, e consequentemente, a quantidade de nós tende a infinito, a solução obtida converge para a solução exata do problema.

Neste trabalho, uma análise numérica é realizada do cordão de solda isolado e sobreposto depositado no aço SAE 1020, pelo processo de soldagem MIG/MAG (Metal Inert Gas/Metal Active Gas). As simulações numéricas foram realizadas com o software ANSYS®, considerando uma fonte de calor em movimento com distribuição do tipo Gaussiana, conforme diferentes autores [14-17], transferência de calor por convecção e radiação nas superfícies e propriedades do material dependente da temperatura para simulações as térmicas, e teve como objetivo avaliar o efeito da sobreposição dos ciclos térmicos de soldagem sobre a região ZAC, através de perfis de temperatura durante a simulação numérica da ZAC utilizando o método dos elementos finitos (MEF). O modelo do fluxo de calor em soldagem foi baseado no trabalho de Goldak [13].

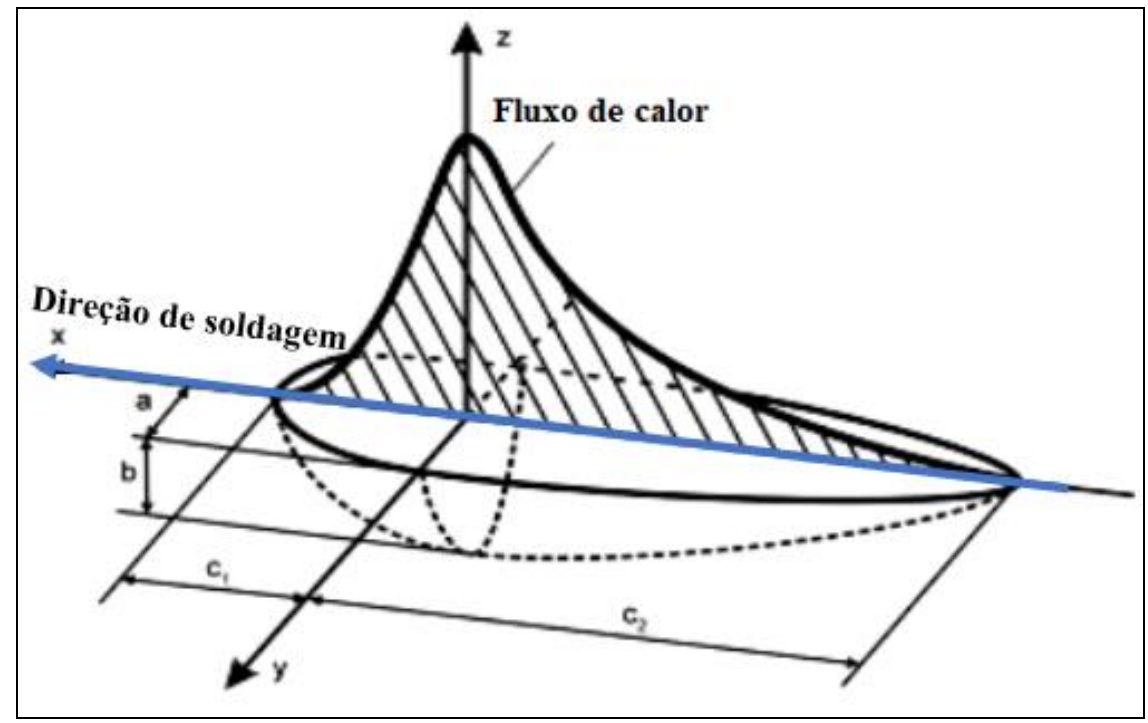

Figura 2: Modelo dupla elipsóide [12].

\section{PROCEDIMENTO EXPERIMENTAL}

As soldagens foram realizadas com o processo $\mathrm{MIG} / \mathrm{MAG}$ convencional com gás de proteção $\mathrm{CO}_{2}$ puro a uma vazão de 15 1/min, com o auxílio de um sistema mecanizado de deslocamento da tocha, sobre o aço carbono SAE 1020, de dimensões 10 × 30 × 100 mm, o metal de adição utilizado foi o arame AWS ER70S-6, 1,2 mm de diâmetro, a distância bico de contato peça (DBCP) utilizada foi $14 \mathrm{~mm}$. A Tabela 1 apresenta os parâmetros de soldagem utilizados na deposição dos cordões de solda. O aporte térmico foi calculado de acordo com a Equação 1.

$$
\mathrm{H}=(\mathrm{U} . \mathrm{I} / \mathrm{v}) \eta
$$

onde:

$H$ é o aporte de calor $(\mathrm{J} / \mathrm{mm})$, 
$U$ é a tensão aplicada $(\mathrm{V})$,

I é a corrente elétrica (A),

$v$ é a velocidade de soldagem $(\mathrm{mm} / \mathrm{s})$,

$\eta$ é a eficiência térmica do processo de soldagem (valor de 0,8 - usual para MIG/MAG).

Foi realizada a sobreposição de cordões isolados, já que o objetivo do trabalho era o efeito da sobreposição dos ciclos térmicos na microestrutura da ZAC do primeiro passe. Após a análise macrográfica, os resultados experimentais foram comparados aos resultados da análise numérica, com o intuito de validar a metodologia computacional.

Após a soldagem, a parte central dos corpos de prova foi extraída para a realização da análise macroestrutural, pois que nessa região as características térmicas da soldagem já se encontram constantes, temperatura máxima. Os corpos de provas foram lixados até a granulometria 600 mesh e posteriormente atacados com nital 5\% para revelar a macroestrutura da solda. Para medição das dimensões dos cordões de solda, utilizou-se o software ImageJ.

Tabela 1: Parâmetros de soldagem utilizados para cada corpo de prova.

\begin{tabular}{|c|c|c|c|c|c|c|c|}
\hline \multirow{2}{*}{$\mathrm{CP}$} & \multicolumn{2}{|c|}{ VS (cm/min) } & \multirow[t]{2}{*}{$\mathrm{VA}(\mathrm{m} / \mathrm{min})$} & \multirow{2}{*}{$\mathbf{U}(\mathbf{V})$} & \multirow{2}{*}{$I(A)$} & \multicolumn{2}{|l|}{$\mathrm{H}(\mathrm{J} / \mathrm{mm})$} \\
\hline & $1^{\circ}$ Passe & $2^{\circ}$ Passe & & & & $1^{\circ}$ Passe & $2^{\circ}$ Passe \\
\hline $\mathrm{S} 1$ & 30 & - & \multirow{6}{*}{5} & \multirow{6}{*}{24} & \multirow{6}{*}{170} & 652,8 & - \\
\hline $\mathrm{S} 2$ & 40 & - & & & & 489,4 & - \\
\hline S3 & 50 & - & & & & 391,8 & - \\
\hline D1 & 30 & 15 & & & & 652,8 & 1350,6 \\
\hline D2 & 40 & 20 & & & & 489,4 & 989,1 \\
\hline D3 & 50 & 25 & & & & 391,8 & 777,1 \\
\hline
\end{tabular}

\section{RESULTADOS E DISCUSSÕES}

A modelagem numérica começa com a especificação da geometria da malha, Figura 3. Para que a análise numérica do modelo construído fornecesse um campo de maior precisão da temperatura e gradiente térmico foi utilizado elementos finitos com malha fina na proximidade da fonte de energia térmica e na parte onde está localizada a poça de fusão, Figura 3b, já na região mais afastada do centro da solda os elementos finitos foram maiores, pois o gradiente térmico é menor e com menor sensibilidade [14, 16, 18$]$.

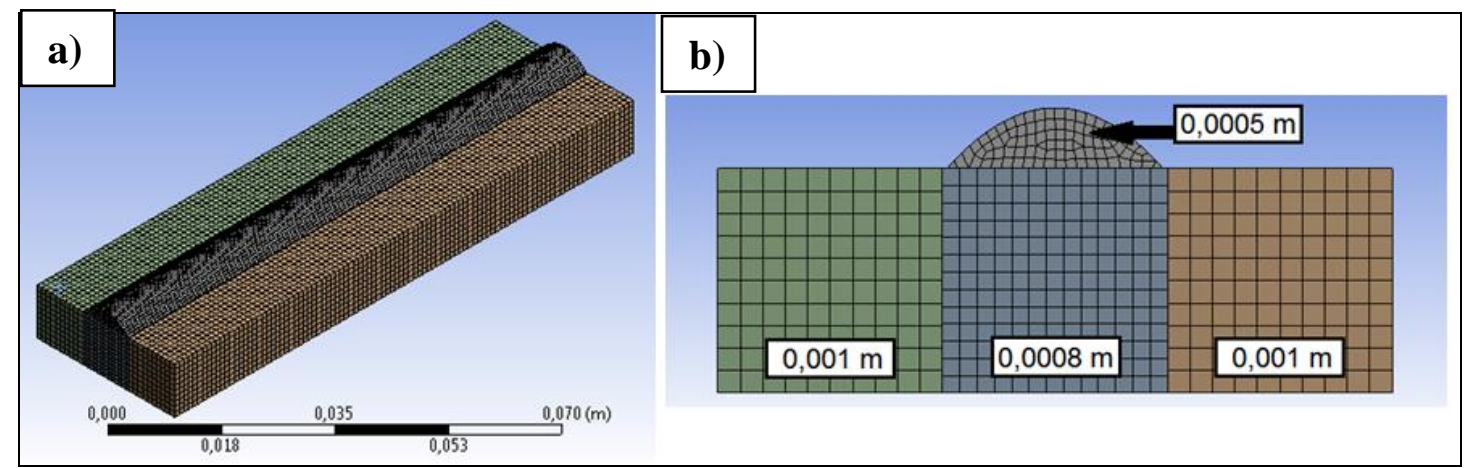

Figura 3: Malha de elementos finitos, a) cordão isolado e b) seção transversal com diferentes dimensões de malha.

Os cálculos de simulação numérica subsequentes do campo de temperatura foram realizados com elementos finitos SOLID90 utilizados na malha, estes elementos térmicos possuem apenas um grau de liberdade em cada nó (temperatura). O material de aço SAE 1020 foi considerado para as simulações, as propriedades físicas (densidade, condutividade térmica e calor específico) do metal de base com temperatura variável foram usadas para análise numérica. Estes valores foram retirados do Metals Handbook Volume 1 [19]. Utilizou-se o mesmo aço SAE 1020 tanto para o metal de base quanto para o metal de adição, já que a composição química, propriedades físicas e mecânicas de SAE 1020 e do arame AWS ER 70S-6 são aproximadas. Nota-se que os dados obtidos referentes à condutividade térmica e o calor específico do aço SAE 1020 vari- 
am de acordo com a temperatura, Figura 4. A densidade pouco variou em função da temperatura, portanto, tal propriedade foi considerada constante, com valor de $7876 \mathrm{~kg} / \mathrm{m}^{3}$.

a)

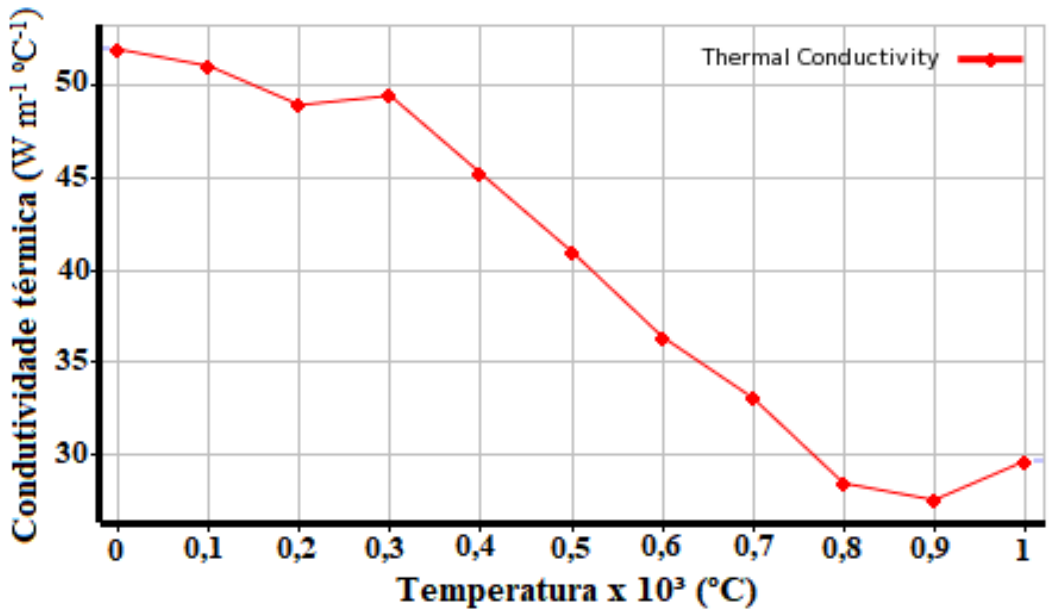

b)

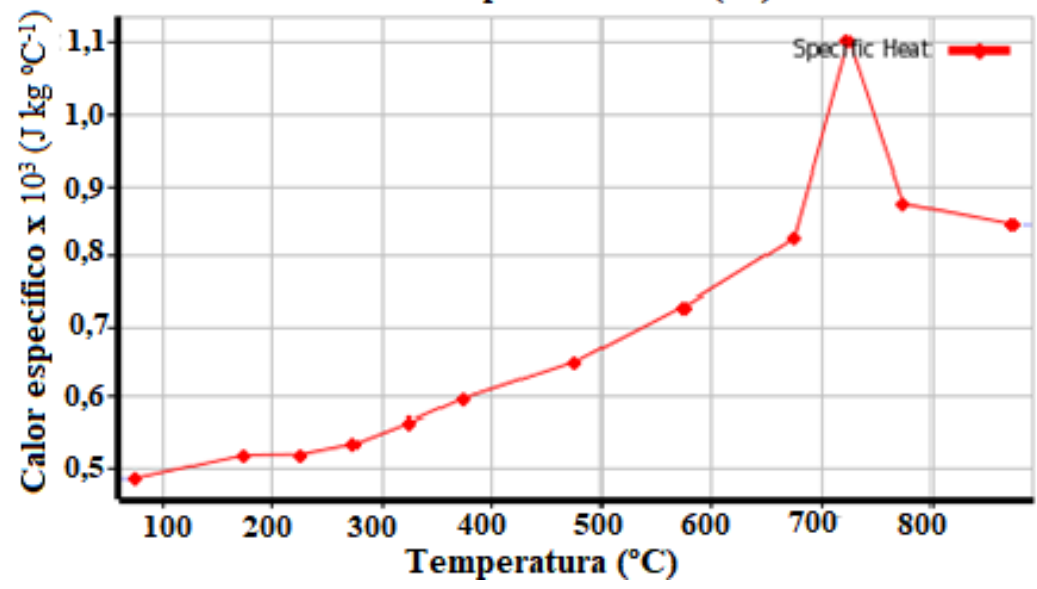

Figura 4: Propriedades físicas do aço SAE 1020 em diferentes temperaturas. a) condutividade térmica e b) calor específico.

Para a análise térmica, os dados determinados experimentalmente foram utilizados para calibrar as fontes de calor da simulação. Para o ajuste do campo de temperatura, a seção transversal do cordão de solda simulado foi correlacionada com o tamanho e a forma da poça de fusão experimental. Os parâmetros das fontes de calor foram otimizados a partir do experimento de soldagem, Tabela 2, conforme representado na Figura 2.

Tabela 2: Parâmetros de soldagem e dupla elipsóide utilizados nas simulações.

\begin{tabular}{l|l|l|l|l|l|l}
\hline \multirow{2}{*}{ CP } & \multicolumn{2}{|l|}{ VS (m/min) } & \multicolumn{4}{l}{ PARÄMETROS DUPLA ELIPSÓIDE (mm) } \\
\cline { 2 - 7 } & $1^{\circ}$ Cordão & $2^{\circ}$ Cordão & a & b & cf & cr \\
\hline S1 & 0,30 & - & 4,80 & 2,70 & 0,60 & 1,60 \\
\hline S2 & 0,40 & - & 4,30 & 2,60 & 0,60 & 1,60 \\
\hline S3 & 0,50 & - & 4,15 & 2,10 & 0,60 & 1,60 \\
\hline D1 & 0,30 & 0,15 & 5,75 & 5,06 & 0,60 & 1,60 \\
\hline D2 & 0,40 & 0,20 & 5,50 & 4,95 & 0,60 & 1,60 \\
\hline D3 & 0,50 & 0,25 & 5,00 & 3,74 & 0,60 & 1,60 \\
\hline
\end{tabular}

Para executar simulações precisas e detalhadas de elementos finitos, as propriedades térmicas do material devem ser conhecidas em altas temperaturas. Uma das vantagens do emprego da liga SAE 1020 foi a 
disponibilidade de propriedades completas do material no banco de dados SOLID90, incluindo temperaturas de transformação da fase metalúrgica, parâmetros do modelo metalúrgico e propriedades termofísicas dependentes da temperatura.

A variação de temperatura prevista numericamente ao longo do cordão de solda é mostrada na Figura 5, além das isotermas características da poça de fusão. É possível observar que não houveram anomalias durante a soldagem, como oscilações ou deslocamento inadequado da fonte de calor, podendo assim concluir que a modelagem do processo se encontra adequada à literatura [20-22].
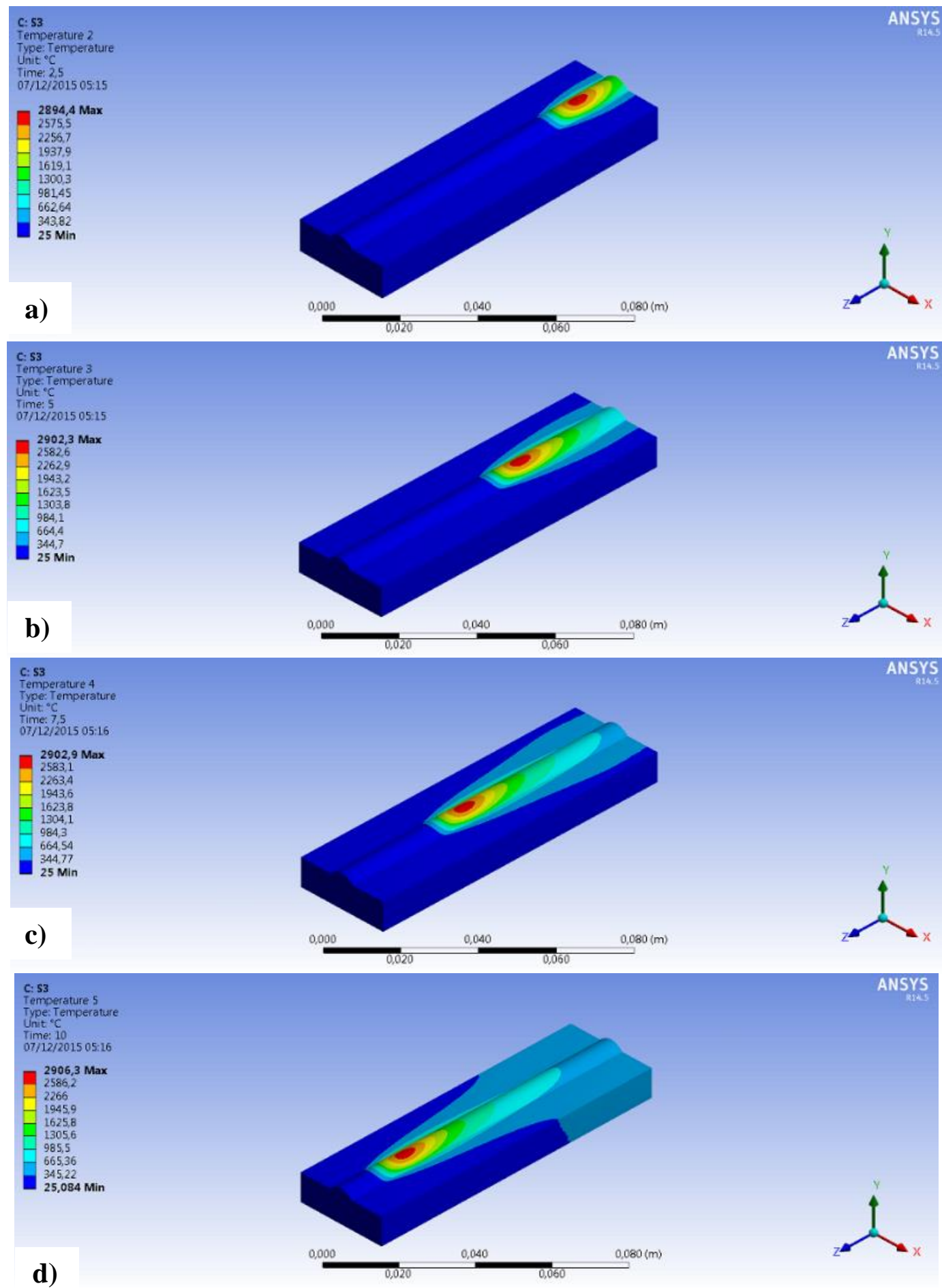

Figura 5: Evolução do campo de temperaturas durante soldagem do SAE 1020, a) 2,5 s, b) 5 s, c) 7,5 s, e d) $10 \mathrm{~s}$.

$\mathrm{Na}$ Figura 5 a análise numérica revelou um alto gradiente de temperatura no metal de solda, onde a 
fusão ocorre por aquecimento do arco elétrico, enquanto que para pontos mais afastados do centro do cordão de solda, o valor de pico do histórico de temperatura diminui. Quando o processo de soldagem é concluído, a amostra soldada é resfriada gradualmente até a temperatura ambiente. As temperaturas características, ou seja, $1300,3{ }^{\circ} \mathrm{C}, 981,45^{\circ} \mathrm{C}$ e $662,4{ }^{\circ} \mathrm{C}$, indicam as temperaturas delimitadoras para a fusão, o crescimento dos grãos (GGZAC), $A c_{3}$, região de grão fino ZAC, GFZAC, e linha $A c_{1}$, abordagem similar foi apresentada por alguns pesquisadores [14,17]. O limite da zona fundida e o limite da ZAC foram identificados a partir da macrografia, Figura 6, e comparados com as linhas isotérmicas correspondentes às temperaturas máximas previstas de $1300,3{ }^{\circ} \mathrm{C}$ e $662,4{ }^{\circ} \mathrm{C}$, respectivamente, mostrando boa concordância entre experimento e modelagem. Observa-se que o modelo numérico representou o modelo experimental acima de $90 \%$ mostrando sua validação, Tabela 3, conforme sugerido por Caron et al. [23]. Os resultados apresentados na Tabela 3 revelam que a dimensão da ZAC é influenciada diretamente pela energia de soldagem.

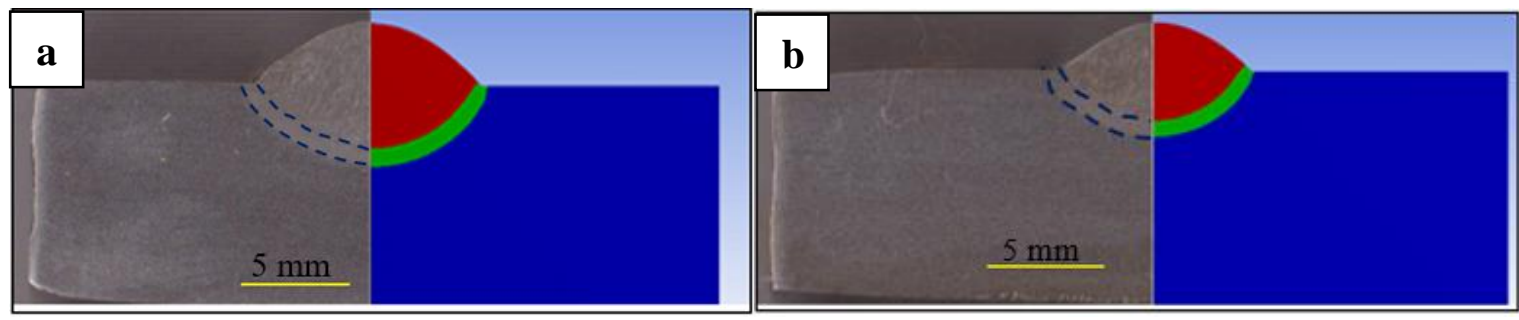

Figura 6: Macrografias das análises experimental e numérica dos cordões de solda simples deposição, a) Amostra S1 e b) Amostra S3.

Tabela 3: Valores médios das dimensões da ZAC para as análises experimentais e numéricas, cordão isolado.

\begin{tabular}{l|l|l|l}
\hline CP & ZAC EXPERIMENTAL $(\mathbf{m m})$ & ZAC NUMÉRICA $(\mathbf{m m})$ & DIFERENÇAS ZACs \\
\hline S1 & $0,71 \pm 0,08$ & $0,74 \pm 0,06$ & $3,00 \%$ \\
\hline S2 & $0,59 \pm 0,05$ & $0,63 \pm 0,07$ & $6,70 \%$ \\
\hline S3 & $0,55 \pm 0,07$ & $0,60 \pm 0,04$ & $9,10 \%$ \\
\hline
\end{tabular}

A partir da Figura 7, pode-se observar que os resultados numéricos e experimentais sob os mesmos parâmetros estavam em boa correspondência, uma concordância satisfatória na sobreposição da ZAC (dimensão) resultante do segundo ciclo térmico de soldagem. A maior divergência entre a análise experimental e numérica foi da amostra D3, Tabela 4. Nota-se, por meio das análises numéricas, que a região de grãos grosseiros da ZAC poderá ser refinada pelo ciclo térmico do segundo cordão, Figura 7.

Na Figura 7 pode se observar que maior fração de revenido estará localizada em uma região relativamente distante da zona de ligação, pois próximo a essa região a alta temperatura atingida pode levar a um aumento do tamanho de grãos, conforme observado por Sun et al. [14]. Esse resultado reforça que a análise numérica pode ser empregada com sucesso no estudo do refino dos grãos grosseiros da ZAC a na técnica de dupla camada. A Tabela 4 apresenta os valores médios das dimensões da ZAC das análises experimentais e numéricas para a solda em simples e dupla deposição. Diferentes trabalhos [14, 16, 18, 24] também revelaram a eficácia do emprego do método de elementos finitos na avaliação microestrutural do metal de solda e na quantificação das tensões residuais em soldagem multi-passes, com erro médio abaixo de $20 \%$.

Para Liu e Qi [25], embora na simulação numérica da deposição de um cordão de solda sobre um anterior a região seja remodelada é impossível descrever a assimetria na seção transversal ao longo do cordão, devido as variações na superfície do cordão de solda ocasionadas pela operação de soldagem. Esse comportamento contribui para um desvio entre os valores numéricos e experimentais de soldas multipasses.

De acordo com Sun et al. [26] apesar das inúmeras tentativas de obter repetibilidade no processo de soldagem em seus trabalhos, existem diferenças perceptíveis nos perfis de solda para as chapas soldadas com um único passe em relação as chapas soldadas com dois e três passes. Tais diferenças influenciam na avaliação da precisão da previsão, isto é, a concordância entre os limites previstos e observados das zonas fundidas e ZAC, em chapas com soldadas com multipasses. 

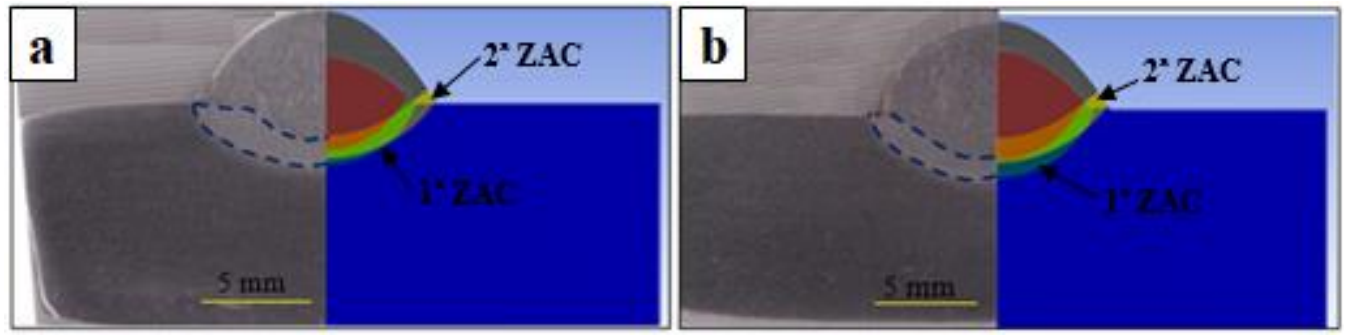

Figura 7: Macrografias das análises experimental e numérica dos cordões de solda dupla deposição, A) Corpo de prova D1, B) Corpo de prova D2.

Tabela 4: Valores médios das dimensões da ZAC para as análises experimentais e numéricas. Soldagem com passes sobrepostos.

\begin{tabular}{l|l|l|l}
\hline CP & ZAC EXPERIMENTAL $(\mathbf{m m})$ & ZAC NUMÉRICA $(\mathbf{m m})$ & DIFERENÇAS ZACs \\
\hline D1 & $1,25 \pm 0,11$ & $1,20 \pm 0,14$ & $4,20 \%$ \\
\hline D2 & $1,35 \pm 0,23$ & $1,32 \pm 0,29$ & $2,27 \%$ \\
\hline D3 & $1,15 \pm 0,14$ & $1,05 \pm 0,18$ & $9,52 \%$ \\
\hline
\end{tabular}

Para Heinze et al. [27], a geometria experimental complexa de soldas é difícil de modelar pelas atuais formulações equivalentes de fontes de calor (3D-Gauss e dupla elipsóide Goldak), especialmente quando calibradas manualmente. A forma da poça de fusão é a principal consequência da configuração limitada da fonte de calor e a principal contribuição para os desvios ocorridos na área da seção transversal do cordão de solda. A geometria experimental da poça de fusão não é estável como a isotérmica simulada no estado quase estável do processo de soldagem. Os autores também relatam que as mudanças na zona fundida e ZAC experimental é ocasionada porque a distribuição de calor muda na direção da soldagem devido a variação da condutividade térmica com temperatura, como mostrado na Figura. 4a.

\section{CONCLUSÕES}

O estudo detalhado neste trabalho utilizou uma metodologia baseada na técnica de elementos finitos e no modelo de fonte de calor gaussiano para simular soldas passe isolado e passes sobrepostos sobre chapas de aço SAE 1020, usando o processo convencional MIG/MAG. Os resultados numéricos de campos térmicos para o estudo de caso foram comparados com os obtidos nos experimentos desenvolvidos no laboratório de soldagem.

A metodologia aqui proposta apresentou uma boa convergência com os resultados experimentais, tanto para soldagens com passe isolado como para passes sobrepostos, os erros percentuais na maioria dos testes ficaram abaixo de $10 \%$.

$\mathrm{Na}$ análise térmica, o formato da zona de fusão obtido concordou numericamente com o experimental, com diferenças variando de 2,3 a 9,5\% ao longo do limite da zona de fusão.

A geometria da poça de fusão e a condutividade térmica constante, além da entrada de calor, influenciam fortemente os resultados numéricos. Assim, é necessário validar cuidadosamente o campo de temperatura de uma simulação numérica em relação à geometria e ciclos térmicos da poça de fusão.

Os resultados revelaram que a correta sobreposição dos passes é fundamental para o sucesso no efeito de refinamento dos grãos da ZAC, dessa maneira é importante considerar a deformação causada na peça devido ao ciclo de soldagem. Essas podem influenciar de forma significativa os resultados também na análise numérica.

Os resultados apresentados neste trabalho revelaram que essa metodologia é uma boa proposta para modelar o processo MIG/MAG para aço carbono. Além disso, é uma excelente ferramenta auxiliar para investigar e projetar estruturas soldadas. Agradecimentos

\section{AGRADECIMENTOS}

Os autores expressam os seus agradecimentos a Faculdade de Engenharia Mecânica da UFPA, Campus Tucuruí, por apoiar a realização deste trabalho. 


\section{BIBLIOGRAFIA}

[1] KOU, S., Welding Metallurgy, 2.ed., New Jersey, John Wiley and Sons, 2003

[2] POORHAYDARI, K., PATCHETT, B. M., IVEY, D. G., "Estimation of Cooling Rate in the Welding of Plates with Intermediate Thickness", Welding Journal, pp. 149-155, Oct. 2005.

[3] WAINER, E., BRANDI, S. D., MELLO, F. D. H., Soldagem - Processos e metalurgia, 4.ed., São Paulo, Edgard Blucher Ltda, 2004.

[4] BHADESHIA, H., HONEYCOMBE, R., Steels: Microstructure and Properties, 4.ed., Cambridge, Butterworth-Heinemann, 2017.

[5] ALORAIER, A.S., IBRAHIM, R.N., GHOJEL, J., "Eliminating post-weld heat treatment in repair welding by temper bead technique: role bead sequence in metallurgical changes", Journal of Materials Processing Technology, v. 153, n. 1, pp. 392-400, Oct. 2004.

[6] RATHOD, D.W., FRANCIS, J.A., ROY, M.J., et al., "Thermal cycle-dependent metallurgical variations and their effects on the through-thickness mechanical properties in thick section narrow-gap welds", Materials Science and Engineering: A, v. 707, pp. 399-411, Nov. 2017.

[7] ALORAIER, A., IBRAHIM, R., THOMSON, P., "FCAW process to avoid the use of post weld heat treatment", Int. J. Press. Vessel. Pip., v. 83, n. 5, pp. 394-398, May. 2006.

[8] LANT, T., ROBINSON, D.L., SPAFFORD, B., et al., "Review of Weld Repair Procedures for Low Alloy Steels Designed to Minimize the Risk of Future Cracking", International Journal of Pressure Vessels and Piping, v. 78, pp. 813-818, 2001.

[9] GARCIA, D. N., FERRARESI, V. A., MOTA, C. A. M., "Evaluation of double-layer weld deposition technique on ASTM A182 F22 steel without post-weld heat treatment", Journal of the Brazilian Society of Mechanical Sciences and Engineering. v. 41, pp. 313-326, Jul. 2019.

[10] FARIAS, R. M., TEIXEIRA, P.R.F., ARAÚJO, D. B., "Thermo-mechanical analysis of the MIG/MAG multi-pass welding process on AISI 304L stainless steel plates", Journal of the Brazilian Society of Mechanical Sciences and Engineering, May 2016.

[11] ROSENTHAL, D., "Mathematical theory of heat distribution during welding and cutting", Welding Journal, v. 20, n. 5, pp. 220-234, 1941.

[12] GOLDAK J. A., CHAKRAVARTI A., BIBBY, M., "A finite element model for welding heat sources", Metallurgical Transactions B, v. 15B, pp 299-305, Jun. 1984.

[13] GOLDAK, J. A., AKHLAGHI, M. Computational Welding Mechanics. Springer, 2005.

[14] SUN, Y.L., OBASI, G., HAMELIN, CJ., et al., "Characterisation and modelling of tempering during multi-pass welding”, Journal of Materials Processing Technology, v. 270, pp. 118-131, Feb. 2019.

[15] XU, D., LIU, X. S., WANG, P., et al., "New technique to control welding buckling distortion and residual stress with non-contact electromagnetic impact", Science and Technology of Welding and Joining, v. 14, pp. 753-759, Jun. 2009.

[16] CHEN, L., MI, G., ZHANG, X., WANG, C., "Numerical and experimental investigation on microstructure and residual stress of multi-pass hybrid laser-arc welded 316L steel”, Materials and Design, v. 168. Feb. 2019.

[17] ANDUD, D., AZHAR, MIM., ZAINUL, WAFW., et. al., "Simulation Study of Welding Distortion on Multi pass Welding of the Fillet Joint", Journal of Science \& Technology, v. 03, n. 01, pp. 1-5, Jan. 2016.

[18] FARAJPOUR, M., RANJBARNODEH, E., "Finite Element Simulation of Welding Distortion in Dissimilar Joint by Inherent Deformation Method", Soldagem \& Inspeção, v. 23, n. 1, pp.60-72, Mar. 2018.

[19] ASM., Metals handbook, Proprieties and Selection of Metals. v. 1, 1993.

[20] KONG, F., MA, F., KOVACEVIC, R., "Numerical and experimental study of thermally induced residual stress in the hybrid laser-GMA welding process", Journal of Materials Processing Technology, v. 211, n. 6, pp. 1102-1111, Jun. 2011.

[21] CASTRO, JA., OLIVEIRA, EM., ALMEIDA, DSS., et al., "Effects of Local Heat Input Conditions on the Thermophysical Properties of Super Duplex Stainless Steels (SDSS)", Materials Research, 2017.

[22] CHO, D.W., CHO, W.I., NA, S.J., "Modeling and simulation of arc: Laser and hybrid welding process", Journal of Manufacturing Processes, v. 16, n. 01, pp. 26-55, Jan. 2013.

[23] CARON, J., HEINZE, C., SCHWENK, C., RETHMEIER, M., BABU, S.S., LIPPOLD, J., "Effect of Continuous Cooling Transformation Variations on Numerical Calculation of Welding-Induced Residual 
Stresses”, Welding Journal, v. 89, pp. 151-160, Jul. 2010.

[24] ZHAN, X., LIU, X., WEI, Y., OU, W., et al., "Numerical simulation on backward deformation of MIG multi-layer and multi-pass welding of thick Invar alloy", The International Journal of Advanced Manufacturing Technology, v. 92, n. 01, pp. 1001-1012, Mar. 2017.

[25] LIU, Z., QI, H., "Mathematical modeling of crystal growth and microstructure formation in multi-layer and multi-track laser powder deposition of single-crystal superalloy", Physics Procedia, v. 56, pp. 411-420, 2014.

[26] SUN, Y.L., OBASI, G., HAMELIN, CJ., et al., "Effects of dilution on alloy content and microstructure in multi-pass steel welds", Journal of Materials Processing Technology, v. 265, pp. 71-86, 2019.

[27] HEINZE, C., SCHWENK, C., RETHMEIER, M., "Effect of heat source configuration on the result quality of numerical calculation of welding-induced distortion", Simulation Modelling Practice and Theory, v. 20, pp. 112-123, 2012.

\section{ORCID}

Douglas Neves Garcia Rafael Albino Bernardi Adry Kleber Ferreira de Lima https://orcid.org/0000-0002-1292-2139

https://orcid.org/0000-0003-2221-5021

https://orcid.org/0000-0003-4150-8903 